\section{THE TRANSIT OF VENUS}

$W E$ have received the following list of selected stations for the observation of the coming transit of Ventc, with the observers appointed to each, according to the arrangements, so far definitive, made by the executive committee of the Royal Society acting under the authority of the Treasury, and also the instructions prepared for the guidance of the observers.

For Retarded INgress and Accelerated Egress Famaica-Dr. Copeland, Capt. Mackinlay, R.A., Mr. Maxwell Hall.

Barbados-Mr. Talmage, Lieut, Thomson, R.A.

Bermuda-Mr. J. Plummer, Lieut. Neate, R.N.

(The Canadian Government will have three observers with six-inch instruments, besides others with smaller telescopes. These observers are acting in direct concert with the British Expeditions).

\section{ACCELERATED INGRESS}

Cape Observatory-Mr. David Gill (H.M. Astronomer), Mr. Maclear, 2nd assistant.

Montague Road, Cape Colony-Mr. W. H. Finlay (Ist assistant at the Cape), Mr. Pett.

Aberdeen Rrad, Cape Colony-Mr. Burton, Mr. C. M. Stevens.

Madagascar-Father Perry, Father Sidgreaves, Mr. Carlisle.

Durban, Natal-A telescope has been provided by the colonists. Mauritius-Mr. Meldrum.

\section{RETARDED EgRESS}

New Zealand-Lieut.-Col. Tupman, R.M.A., Lieut. Coke, R.N. Brisbane-Capt. Morris, R.E., Lieut. Darwin, R.E., Mr. Peek. Melbourne-Mr. Ellery and staff.

Sydney-Mr. Russell and staff.

\section{INSTRUCTIONS TO OBSERVERS}

The "Instructions issued by the International Conference on the Transit of Venus, I882," are recommended for general adoption. These instructions are, in a great measure, founded upon "Strggestions for a Draft of Instructions for the Observers," which the English Committee circulated for consideration in October, 188r. But as copies of the Instructions, issued by the International Conference, may not reach observers in the English colonies who may be willing and able to co-operate effectively, and as there are some additional explanations and cautions which the English Committee consider it desirable to give their observers, the following instructions have been issued. It is most earnestly hoped that all observers who are able to cooperate effectively in the observation of contacts will, whatever else they may observe, observe the contacts as defined; and that they will employ sufficient magnifying powers on their telescopes and use a field of view of moderate brightness. These are essentials, if their observations are to be combined with the results of the English expeditions.

I. It is most important that the apertures of the refractors used for the observations of the contacts should be nearly equal. The apertures of the teleseopes available for the English expeditions are not generally larger than six inches; and it is therefore recommended that observers with larger apertures should stop them down to six inches, but not reduce them below that size. It is considered that perfect instruments of four inches aperture may give results sufficiently comparable with those made with the six-inch telescopes to allow all the observations to be combined in one common discussion; and it is hoped that observations with such instruments will be made. Observations with good instruments of smaller apertures than four inches will be exceedingly valuable for combination with others made with instruments of the same class, provided that powers of 100 to I 50 can be employed on them. Observation of contact made with very low pozvers are useless for the objects in view. When reflectors are used, apertures should not be less than seven inches.

2. The observers are requested to furnish tests of the optical performances of their telescopes, such as-

(I) The appearances presented by the disc of a bright star when the eye-piece is pushed within and pulled without the focus.

(2) The power of the telescope to separate some well-known close double stars.

(3) Whether the observer was able to see the "rice grains" or "granulations" on the sun's disc on the day of the transit and if not, whether he is generally able to see them with the same instrument and a power of 150 on days of good definition. 3. The observers are recommended to employ a first-surface reflecting prism to diminish the sun's heat and danger to the observer's eye, and a compensated neutral-tint wedge between the eye-piece and the eye.

4. The eye-pieces recommended are the "negative," or a "Steinheil's simple achromatic positive eye-piece." When the latter is used, two pairs of very fine parallel wires should be placed on opposite sides of the field of view at distances corresponding to a second of arc apart. Such wires are useful in judging of the brightness of the field of view, and of the sufficiency of the optical power employed to subdivide a second of arc into tenths, and in estimating the angular separation of the limbs in descriptions of any phenomena which may be seen near the contact.

5. All attempts to observe the contacts with double-image eyepieces are to be avoided.

6. The use of Dawes' solar eye-piece is not recommended. This eye-piece is exceedingly valuable for the examination of small detached portions of the solar disc; but the field of view is very limited, and, if clouds were passing, there would be practical difficulties in keeping the point of contact exactly in the centre of the field, whilst the effects of the stop would certainly be injurious to the contact observations near the edge of the field.

7. The magnifying power should be about I50, and, even if the definition is not good, this power should not be much reduced. It is essential that the observer should be able to subdivide a second of arc into tenths, and to do this a high power is necessary.

8. It is extremely desirable that all the observations of contact should be made in fields of view of nearly the same brightness, and the illumination should neither be one of extreme brightness, which greatly complicates the phenomena seen near the contacts from the increased effects of irradiation, nor one so faint that difficulties may be experienced in distinguishing changes in the jllumination of the sun's limb near the point of contact, which can only be recognised by contrast. If pairs of very fine spider webs are placed at a distance corresponding to a second of arc in the focus of a positive eye-piece, they may be used for the determination of a suitable illumination of the field of view by observing at what part of the neutral-tint wedge the wires can just be seen with sufficient distinctness to allow the observer, without difficulty, to sub divide the interval between them into tenths; but in the application of the test the wedge must be shifted quickly, and the eye not allowed to strain itself in attempting to separate the wires, or a too dull field of view will be secured. The test is a delicate one to apply, and it is probably better to replace it in practice by the following:- When the sun is free from clouds, determine with what parts of the neutral-tint wedge the sun's limb can just be observed with comfort, and with what part of the wedge the $\operatorname{limb}$ can just be distinctly and clearly seen. A mean portion of the wedge between these extremes should be adopted as a standard field of brightness with a clear sun; and this degree of brightness, learnt by practice as a habit of the eye, should be adopted for the brightness of the field of view in the observation of the contacts.

9. The expressions "contact," "apparent contact," "actual contact," "real contact," and "true contact," appended to time records without any explanation of the sense in $\mathbf{v}$ hich the word "contact" is used, are liable to be misunderstood. Such expressions should not, therefore, be employed, unless a description of the particular kind of contact referred to is appended.

IO. The phenomena seen, by most observers, near the time of contact, in a moderately bright field of view, are of a complex character, and extend over considerable intervals of time. It is therefore necessary to direct the attention of the observers to some distinctive phases which all those, who have sufficient optical means, should certainly see unless prevented by clouds. Subject to the remarks $a, b, c, d$, the times to be recorded near the internal contacts are as follows :-

At Ingress. - "The time of the last appearance of any wellmarked and persistent discontinuity in the illumination of the apparent limb of the sun near the point of contact."

At Egress. - "The time of the first appearance of any wellmarked and persistent discontinuity in the illumination of the apparent limb of the sun near the roint of contact."

(a) The expression "zel. -marked and persistent discor tinuity 
in the illumination of the apparent limb of the sun near the point of contact" is intended to guard observers against giving times for the contacts when there may be a suspicion only of some slight disturbance, haze, shadow, or interference phenomena. It is a point of primary importance that all the observers shall, as far as possible, observe the same kind of contact; and it is therefore desirable that the times recorded for contacts should refer to some marked discontinuity in the illumination of the sun's limb, about which there cannot be a doubt, and which may be supposed to be recognisable by all the observers.

(b.) If a pure geometrical contact, " contact géométrique sans déformation," is alone seen, this is the only time which can be given for the contact; but if haze, shadow, ligament, or black drop is seen, then the last time, when any marked discontinuity in the illumination of the sun's limb near the point of contact is distinctly recognised as independent of mere atmospheric tremior, is a time which should be recorded at ingress; and the first time at which such a marked discontinuity in the illumination is certain, is a time which should be recorded at egress. But if the haze, shadow, ligament, or black drop is ever seen as dark, or nearly as dark, as the outer edge of the planet, the time of greatest blackness, when it is last seen at ingress, or first seen at egress, as dark or nearly as dark as the outer edge of the planet, is also to be mo:t carefully recorded; this phase appears to correspond most nearly to what is given by some observers as "contact géométrique sans déformation," and probably differs but little from what most observers would call "contact," if restricted to a single phase. Near the time of contact the attention of the observer shculd be directed to the parts of the sun's limb near the point of contact. The discontinuity of the illimination of the sun's limb near the point of contact will be recognised by the contrast between the illumination at and on each side of the point of contact.

(c) At ingress the contact can also ke regarded as the time "when light is about to gliwmer all across the dark space between the cusps." For so long as the sunlight has not "glimmered" across the dark space between the cusps, there must be "some well-marked and persistent discontinuity in the illumination of the sun's limb near the point of contact." In this definition the attention of the observer is directed to the light of the cusps which is encroaching upon the "dark space" between them, whilst in the defitition adopted in these instruc tions his attention is directed to the disappearance if the dark space between the cusps which is being encroached upon by the light of the cusps. Great care is, however, required when the contact is thus regarded, that the glimmering of the light of the "auréole," "periumbra," or "sunlight refracted through the atmosphere of Venus," across the dark space between the cusps be not taken for the contact. The time thus recorded n ould be earlier than the contact required by about a minute of time. On the other hand, a time must not be given "when sunlight is dis. tinctly recognised" between the limbs without any direct reference to the time when the dark space between the cu: ps was last recognised.

(d) The observer should clearly and distinctly indicate the times which, in his opinion, correspond most nearly to the contacts as defined above. But in cases where he has any doubt about the second of time which ought to be given, on account of the gradual obliteration and restoration of the illumination of the sun's limb near the point of contact, or on account of any change in the degree of darkness in the haze seen, he may give two times, with a clear intimation of his inability to say which of the two corresponds most nearly to the time of contact; and such observations, if the limits of uncertainty are confined within a few seconds, will be amongst the most satisfactory observations which can be made. The observer must not be discouraged from giving the nearest second possible on account of the lingering character of the contact. The change in the angular separation of the limbs of Venus and the sun is only a tenth of a second of arc in about two seconds of time. It is with seconds of arc, and not seconds of time, that we are ultimately concerned; and one tenth of a second of arc is a very small quantity to be measured on the sun's limb. In the Transit of 1874, when, however, the change in the angular separation was much slower than in I882, many observers were discouraged and disappointed at the degree of accuracy attainable, and the observations appear to have suffered from a feeling on the part of the observers that such observations as they could make were worthless. This feeling should be carefully guarded against.

II. It is hoped that all the observers may be able to observe contacts as defined; but should an observer see a contact which, in his opinion, does not agree with the definition, he must record the time of ccntact, and describe the nature of the contact observed, with drawings to illustrate his meaning. It is desirable that an observer should record the times at which any very distinctive phenomena are first or last seen near the contacts. But the multiplication of unneressary time records near the contacts is, in itself, a serious evil, and should be carefully guarded against ; and, more particularly, records of time corre. sponding to "clear sunlight between the limbs of Venus and the Sun" are to be avoided after all touch, as shown by some recognisable disturbance of the illumination of the sun's limb near the point of contact, has ceased at Ingress between the limbs of Venus and the sun. When this touch has once ceased, all subsequent records of time, unless accompanied by direct measurements of the angular separation of the limbs at these times, afford no possible means of determining the angular separation of the centres of the sun and Venus. Such records are, therefore, of no direct value; but unless great care is taken they may be accep:ed as referring to contacts, and may thus lead to most serious error.

Attention to this point is more particularly necessary when otservations of the contacts are picked up through clouds. If, therefore, times are recorded at "ingress" for "di tinct band of light between the edge of Venus and the sun's limb" or "Venus well on the sun's disc," the observer must most distinctly state whether this time record is intended to mean that the haze, shadow, ligament, or black-drop was certainly seen within a few seconds, at most five, of the recorded time, or whether it is intended merely to state as an isolated fact that the contact was over at the time recorded. Time records of the first class"are valuable, but those of the latter class are useless, and may be misleading.

12. If the limbs of Venus, at internal contact, as defined in Io, fall within the sun's disc, then the observer should give, as accurately as he is able, probably to seven or eight seconds, the time at which the limbs of Venus and those of the sun mentally completed would appear to touch. This observation must be a rcugh one; but it is desirable in the case indicated to give it as a check upon the principal phase observed.

13. External contacts should be observed. The value of the ex ernal and apparent contacts, which are referred to the "visible" or "apparent" limb of the sun, will greatly deperd upon the extent to which uniformity in the instrumental equipments, and in the brightness of the fields of view, may have been secured.

14. It is desirable that all observers who have double-image micrometers : hould measure the cusps at egress, and the distances between the limbs of the sun and Venus after internal contact at ingress; but the eye-pieces should not be changed at ingress until there is a broad band of sunlight between the limbs of Venus and the sun. If an observer feels perfectly confident in his ability to change the double-image micrometer for an ordinary eye-piece and to focus properly, after making cusp measures, before internal contact at ingress, then such an observer may venture to make cusp measures at ingress; but it is most earnestly hoped that observers will not run any risk of losing the internal contact observations at ingress for the sake of these cusp measures. It is necessary, not only for the eye-pieces to be changed and the focus found, but that time should be allowed for the eye to accommodate itself to the new eye-piece before the internal contact takes place, or satisfactory observations of internal contact will not be made.

I5. In all cases the recorded times should be those taken directly from the chronometer or clock used.

I6. The malier's name and number of the chronometer or clock used shculd be given.

17. The errors of the chronometers and clocks should be given for a few days before and after the transit, and a clear statement made of how these errors have been determined. Chronometers should be compared, whenever possible, before and after the transit with some standard cluck.

I8. The greatest care should be taken to insure the accuracy of the entries of times from the clocks ard chronometers used in the contact observations, and unusual care is required in the verification of the minutes and the half-minutes. In most astronomical observations, if the seconds are recorded correctly, 
the minutes can be supplied with perfect certainty by calculation; but in observations of a transit of Venus the contacts from apparent contact to the "last appearance of any marked "disturbance of the illumination" may, in a moderately bright field, extend over more than a minute of time; and if any misconception of the kind of contact which has been observed should be possible from ambiguity in the description given by the observer, then a serious error may be introduced into the discussion of the results from the adoption of a wrong minute and wrong kind of contact for this observation. In the use of chronometers mistakes of half a minute have occasionally been made by taking the "arrow end" instead of the "longer end" of the seconds hand.

In all cases, therefore, such precautions should be taken to verify the minutes and half-minutes that errors of entry can be asserted to be impossible.

I9. Approximate latitudes and longitudes of the station, and the authorities from which they are derived, should in all cases be given, to gether with the local names of the station.

20. The position of the observer should be permanently marked, and, if possible, referred to three or more surrounding natural objects, as mountains, so that the position can be recovered if the mark should be accidentally destroyed.

21. In cases where the errors of the chronometers or clocks and the geographical position of the observers are independently determined, the observations upon which these determinations rest should be given.

22. The descriptions of the contacts which correspond to the time records should be written out by each observer, and entered in an indelible form, before any discussion or comparison of the observatious with those made by any other observer has been made. On no account is a written figure to be altered. On no account is a new figure to be written upon an old one. Any correction is to be written on another line, and attested by the signature of the observer.

23. Copies of these observations, authenticated by the signature of the observer, with the necessary materials for the determination of clock-errors, longitudes, and latitudes, should be forwarded by the next or following mail to the Committee at the Royal Society, Burlington House, London. In the case of the Government expeditions, the original documents must be placed in the hands of the official in charge of the Treasury chest at the station, by whom the originals will be retained until the Committee have acknowledged the receipt of the copies and forwarded instructions for the despatch of the originals.

24. Practice with the artificial models of the transit will be useful to observers as a preparation for the slowne:s with which changes in the appearances piesented near the internal contacts take place. But the exact phases presented in the real transit cannot at present be reproduced in the models, and, unless care is taken, model practice may do more harm than good in leading observers to expect a definite succession of phenomena near the internal contacts which they may be unable to recognise in the actual transit. The complicated phenomena presented near the internal contacts are, no doubt, chiefly due to diffractional irra. diation; but in the case of the models we have the sun and Venus bounded by hard edges. The diffraction phenomena beyond the geometrical boundary of the artificial sun, and the interference phenomena between the limbs of the sun and Venus, are continually changing as the disc, which represents Venus, approaches nearer and nearer the hard edge which represents the geometrical boundary of the sun's disc. These conditions introduce complications into the phenomena seen with the model which have nothing exactly corresponding to them in the real transit; whilst, on the other hand, the presence of the partial illumination of the atmosphere of Venus introduces difficulties in the observation of the real transit which have nothing exactly corresponding to them in the models in ordinary use.

\section{ATOMIC WEIGHTS 1}

SEVEN years after the publication of the first volume of Dalton's "New System of Chemical Philosophy," and therefore at a time when the data from which atomic weights could be deduced were few and inaccurate, Prout I "The Constants of Nature. Part V. A Recalculation of the Atomic Weights." By Frank Wigglesworth Clarke, S. B., Pr. fessor of Chemistry and Physics in the University of Cincinnati. (Washington: Smithsonian Institution, 1882.) promulgated the hypothesis that the atomic weights of all the elements are multiples of that of hydrogen.

This hypothesis was soon shown to be without foundation in fact, but in the modified form given to it by Dumas--viz. the atomic weights of all the elements are whole, half, or quarter multiples of that of hydrogen-it found very considerable acceptance among chemists, although it was strongly opposed by many.

In I860 Stas published the results of very carefullymade determinations of the atomic weights of nitrogen, chlorine, sulphur, potassium, sodium, lead, and silver. Stas concluded from these results that Prout's hypothesis is purely imaginary; that each elementary substance is a distinct entity, and exhibits no simple mass relations with other elements.

Marignac criticised the numbers obtained by Stas, objecting that unless an atomic weight is determined by wholly different series of experiments it cannot be accepted as final, and making the somewhat astonishing statement that possibly the composition of a given compound is not altogether invariable. The reply of Stas appeared in the form of his famous "Nouvelles recherches sur les lois des proportions chimiques, sur les poids atomiques et leur rapports mutuels," wherein the fixity of composition of many compounds was firmly established, and numbers were deduced, from widely different and most carefully conducted series of experiments, for the atcmic weights of silver, iodine, bromine, chlorine, sulphur, nitrogen, lithium, potassium, sodium, and lead, which numbers appeared finally to negative the hypothesis of Prout, even in the form given to it by Dumas.

The experimental work of Stas has been accepted as unimpeachable by every chemist. The "Nouvelles recherches" is a classical work. But in I878 Dumas showed that pure silver, prepared by the method adopted by Stas, gave up weighable quantities of oxygen when heated in vacuo. The numbers given by Stas as the atomic weights of the elements enumerated above may therefore not represent the true atomic weights of these elements. The importance of the discovery made by Dumas is emphasised when we know that the atomic weight of silver is a fundamental number, on which most of the other atomic weights determined by Stas depend.

In 1872 , Crookes communicated to the Royal Society the results of an extremely careful determination of the atomic weight of thallium; the mean number obtained, $203^{\circ} 642$, was regarded by Crookes as strongly against Prout's hypotbesis.

Recent work, physical as well as chemical, has again caused attention to be turned to the hypothesis which would regard the elements as forms of one kind of matter.

The necessity for a revision of many atomic weights has impressed itself on chemists; and several very carefu revisions, notably that of the atomic weight of antimony by Cooke, and of aluminium by Mallet, have recently been made. But in addition to these new data there exist many determinations, which, if properly collected and digested, would be of much importance. Prof. Clarke has done this most admirable service to science.

"Atomic Weight Determinations; a Digest of Inves. tigations published since 1814, " by Prof. G. T. Becker, has already appeared as Part iv. of the Smithsonian Constants of Nature; Prof. Clarke's Recalculation completes the Digest; together these form a contribution to chemical science of the first importance.

The ratio between the atomic weights of oxygen and hydrogen is that first discussed. Each series of experiments is considered separately ; the mean value is found and the probable error of this mean is assigned by the method of least squares. Those elements, the atomic weights of which have been most carefully determined, viz. silver, chlorine, bromine, iodine, potassium, sodium, and sulphur, are next considered.

The discussion of atomic weights involves many chemi- 